\section{Discussion}

External physical characteristics as a means of assessing gestational age were preferred to neurological signs for two reasons. Firstly, the sample was biased towards LBW, and therefore many infants would be attached to intensive care equipment, making impossible a number of tests needed for the neurological examination; secondly, it was felt that neurological signs might be altered by the delivery process and drugs given to the mother.

A number of methodological problems are evident in earlier studies. Prior knowledge of maternal dates has been a serious difficulty in several, where the investigator has both examined the baby and questioned the mother concerning the details of the LMP. Such a dual role inevitably influences the findings: the maternal menstrual data must be assessed by an independent worker.

The use of a correlation coefficient has been common in previous studies but has not been entirely satisfactory due to the paucity of babies of shorter gestations. In the present sample a relatively large number of preterm babies of known gestational ages $(36 / 156)$ was included. For this reason the sample was subdivided into preterm and term LBW groups and analysed separately. The analysis highlights the technical problems of the score. It has not always been appreciated by previous investigators that the precision of the TMS varies with gestational age, since 0.9 points represents a week before 30 weeks and 0.8 points equals a week before 32 weeks, whereas at 39 weeks, 3 points represent a week. The difference in the mean gestational age for preterm infants calculated by the TMS method from that derived from the LMP is 1.5 weeks, compared with 0.4 weeks for infants at term, which shows the inaccuracy of the use of this score in the preterm LBW group

The use of external characteristics seems an easy and satisfactory method of assessing the gestational age of the term baby, and the error is well within the range acceptable in other analyses using complex biological investigations. The results for term infants are remarkably consistent, considering the vagaries of maternal memory and the tacit assumption that ovulation occurs on the 14th day of each cycle. In contrast, the results obtained for preterm infants underline the inaccuracies and uncertainty present in methods currently available for corroborating the maternal data in this group. The small numbers of infants, the effects of illness and drugs, type of delivery, and the structure of the score all militate against accurate assessment in these infants, and further study of this problem is needed.

\section{Conclusion}

For routine clinical purposes the use of the external physical characteristics is a simple and convenient method for estimating gestational age. It is particularly accurate at term but is less precise for babies of short gestation.

I thank Professor John Dennis for reviewing the maternal data, Mrs Phyllis Easton for technical assistance, Professor R. G. Mitchell for his helpful comments, and Action for the Crippled Child for generous financial assistance.

\section{References}

Cater, J. (1978). A population study of LBW infants and their matched controls with special reference to fetal growth. MD thesis, University of Edinburgh.

Dubowitz, L. M. S., Dubowitz, V., and Goldberg, C. (1970). Clinical assessment of gestational age in the newborn infant. Journal of Pediatrics, 77, 1-10.

Farr, V., Kerridge, D. F., and Mitchell, R. G. (1966). The value of some external characteristics in the assessment of gestational age at birth. Developmental Medicine and Child Neurology, 8, 657-660.

Robinson, R. J. (1966). Assessment of gestational age by neurological examination. Archives of Disease in Childhood, 41, 437-447.

Correspondence to Dr John I. Cater, Department of Child Health, Ninewells Hospital and Medical School, Dundee DD1 9SY, Angus.

\title{
Dissolution of bilateral staghorn cystine renal calculi
}

\section{G. RUYSCH VAN DUGTEREN AND J. WIGGELINKHUIZEN \\ Department of Paediatrics, University of Cape Town, and Renal Clinic, Red Cross War Memorial Children's Hospital, Cape Town}

SUMMARY Bilateral staghorn renal calculi in a 7year-old girl with cystinuria were dissolved over a period of 6 months, using a high fluid intake, urinary alkalinisation, and D-penicillamine. Even in children with extensive cystine urolithiasis, medical management may avert the need for surgery.
Cystinuria is an inherited complex autosomal recessive defect in which there is decreased proximal renal tubular reabsorption of filtered cystine, lysine, arginine, and ornithine. Defective transport of these amino-acids in the gut has also been demonstrated, but the clinical symptoms are related only to the associated stone formation (Crawhall and Watts, 
1968). Cystine is the least soluble of all the naturally occurring amino-acids, and crystalluria and stone formation tend to occur when the cystine concentration exceeds the solubility limit of approximately $400 \mathrm{mg} / \mathrm{l}( \pm 1660 \mu \mathrm{mol} / \mathrm{l})$ at $\mathrm{pH} 7 \cdot 0$ and temperature $37^{\circ} \mathrm{C}$ (Macdonald and Fellers, 1968).

The excretion of cystine in quantities large enough to cause stone formation probably only occurs in homozygotes (incidence $\pm 1: 40000$ ) (Dahlberg et al., 1977). The age of presentation and severity of disease appear to be related more to drinking habits and other factors affecting urinary concentration than to the actual quantity of cystine excreted or the genotypic variants of cystinuria.

We report what we believe to be the first case in which bilateral staghorn cystine calculi in a child were completely dissolved by medical means.

\section{Case report}

A 7-year-old girl of mixed ethnic origin presented with recurrent urinary tract infection and haematuria. No member of her family was known to have calculi or other renal problems, but there was an unconfirmed report that the patient had once passed a small stone per urethram. Clinical examination showed no abnormalities. Her urine contained numerous pus cells and red cells, and gave a pure growth of Escherichia coli.

A plain $x$-ray showed the presence of bilateral renal staghorn calculi, with clusters of smaller stones in the calyces (Fig. 1). An intravenous urogram showed mild bilateral caliectasis without

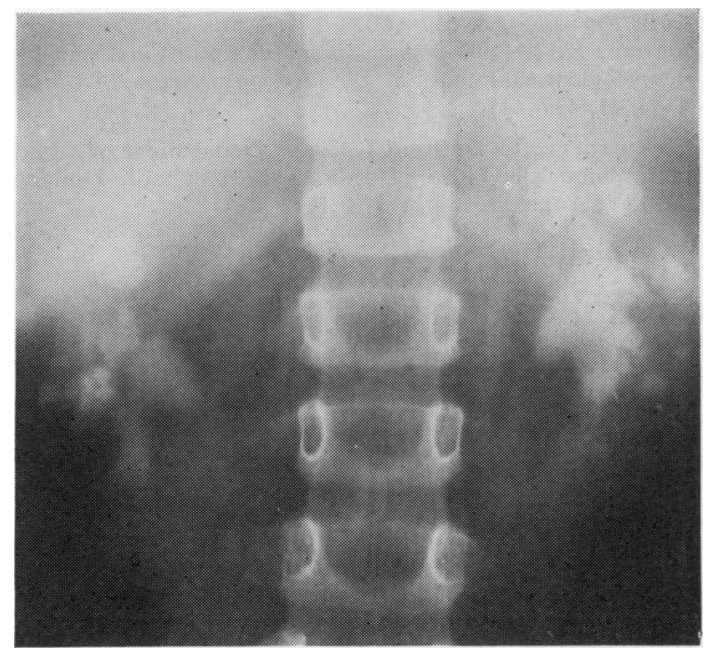

Fig. 1 Renal tomogram before treatment showing bilateral staghorn calculi and multiple clusters of smaller calculi. evidence of obstruction or renal parenchymal damage.

Serum sodium, potassium, urea, creatinine, calcium, phosphorus, alkaline phosphatase, protein, uric acid, and acid-base values were normal, as were urinary $\mathrm{pH}$ and calcium, phosphate, and uric acid excretion values. 24-hour creatinine clearance was $110 \mathrm{ml} / \mathrm{min}$ per $1.73 \mathrm{~m}^{2}$.

Hexagonal crystals typical of cystine were readily demonstrable on microscopical examination of a chilled early morning urine specimen which had been acidified with glacial acetic acid to $\mathrm{pH} 4$. The presence of cystine in the urine was demonstrated by the cyanide-nitroprusside test. High voltage paper electrophoresis confirmed the presence of cystine, and showed abnormally large amounts of the 3 dibasic amino-acids-lysine, arginine, and ornithine. Quantitative amino-acid analysis by ion-exchange column chromatography showed that the patient was excreting $>10$ times the normal maximum quantity of cystine in the urine- $-860 \mu \mathrm{mol}$ per 24 hours compared with the normal adult rate of $<80$ $\mu \mathrm{mol}$ per 24 hours. The lysine, arginine, and ornithine levels were similarly raised, confirming the diagnosis of homozygous cystinuria.

Cystine excretion by both parents and 2 of the patient's 3 siblings was moderately raised (170-340 $\mu \mathrm{mol}$ cystine per 24 hours). Levels of lysine, arginine, and ornithine excretion were also slightly raised in the father and 2 siblings, but none had radiographical evidence of renal calculi. They are therefore presumed to be heterozygotes.

\section{Treatment and progress}

The urinary tract infection was cleared with cotrimoxazole, and prophylactic chemotherapy with nalidixic acid was maintained.

Treatment was directed towards decreasing the concentration and increasing the solubility of cystine in the urine. This was achieved in three ways. Firstly, an increased fluid intake during day and night was encouraged. This effectively doubled the urine output and thus reduced the concentration of urinary cystine. Secondly, by increasing urinary $\mathrm{pH}$ from 6 to $>7 \cdot 5$ the solubility of cystine was increased approximately 2 -fold. $8 \mathrm{~g}$ effervescent sodium citrotartrate was given 4 times a day with the additional fluids and was found to be effective in keeping the urine alkaline. The added sodium load (about 200 $\mathrm{mmol} / 24$ hours) produced no evidence of fluid overload and had a secondary beneficial effect by inducing an osmotic diuresis. Acetazolamide and sodium bicarbonate were ineffective in keeping the urine alkaline for any length of time. Thirdly, D-penicillamine was given. This drug when excreted in the urine 
combines with cystine, forming the much more soluble penicillamine-cysteine complexes. The initial dose was $125 \mathrm{mg}$ twice a day and during 3 weeks this was increased to $250 \mathrm{mg}$ three-times daily.

Once the child had been stabilised on this regimen, she was discharged and seen monthly. She remained asymptomatic and no side effects of D-penicillamine or other treatment were observed. Patient (and parent) compliance was excellent as reflected by the

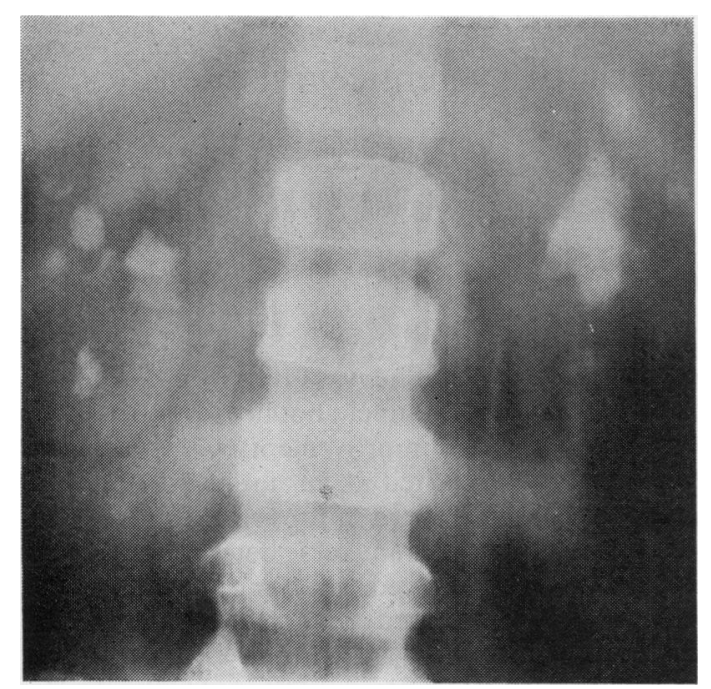

Fig. 2 Renal tomogram after 2 months' treatment, showing reduction in size and number of calculi.

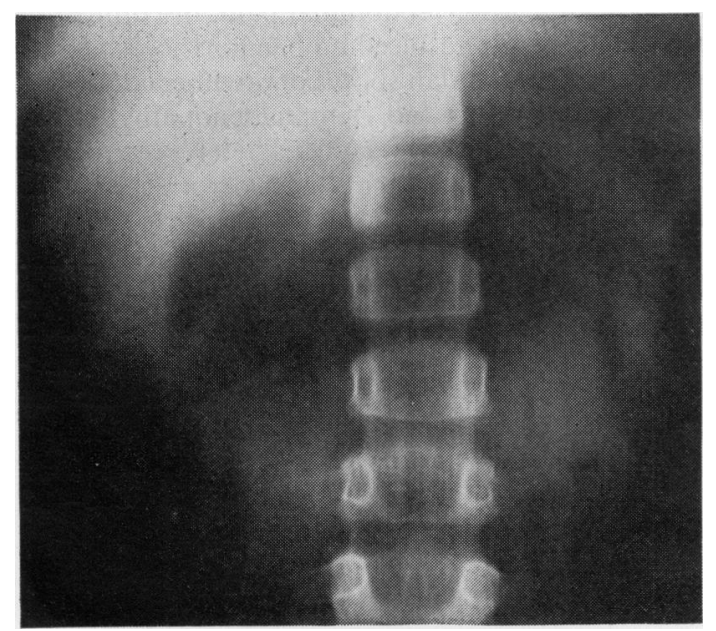

Fig. 3 Renal tomogram after 6 months' treatment, showing no evidence of calculi. passage of large volumes (1800-2300 ml/24 hours) of dilute urine, with a $\mathrm{pH}$ never dropping below $7 \cdot 5$.

Within 3 months of starting treatment the renal calculi were markedly reduced in size and number, and after 6 months these had completely disappeared (Figs 2 and 3). D-penicillamine was then stopped because of the risk of long-term side effects.

\section{Discussion}

Nephrolithotomy was initially considered the prime form of treatment. The infection however was readily controlled and as no evidence of obstruction was found, and renal function was normal, immediate surgery did not seem indicated. In addition, the small but definite risk attendant on bilateral nephrolithotomy, and the fact that total removal of multiple calculi is difficult, as well as the inevitability of scarring of both kidneys which would form a nidus for further calculus formation, led us to attempt to dissolve the calculi.

Failure to dissolve calculi may be due to inadequate treatment or to lack of patient compliance: considerable motivation is required to get a child to take extra fluids, especially in the middle of the night. Calculi may also fail to dissolve either because they are mixed stones containing calcium salts due to coexisting metabolic disorders, such as hyperparathyroidism, or because of obstruction to urine flow.

Although medical management is preferable to surgery, it must be remembered that D-penicillamine toxicity is often a limiting factor (Stokes et al., 1968). An acute hypersensitivity reaction with a transient rash, fever, arthralgia, and lymphadenopathy is common but will often resolve if treatment is temporarily reduced or stopped. More serious complications-such as agranulocytosis, immunecomplex-induced nephrotic syndrome, drug-induced systemic lupus erythematosus, or Goodpasture's syndrome-usually require discontinuation of the penicillamine. Potential pyridoxine deficiency may be avoided by prophylactic pyridoxine therapy.

To prevent recurrence of cystine calculi, patients will need to understand that high fluid intake especially at night, and probably also alkalinisation of the urine, will have to be continued for life. Dpenicillamine is best given only if new calculi are formed (Dahlberg et al., 1977). Less toxic but effective agents, such as $\mathrm{N}$-acetyl penicillamine (Stokes et al., 1968) or mercaptopropyonylglycine (Terhorst and Stuhlsatz, 1975), may become generally available in the near future.

We thank Dr H. Henderson and Dr E. Diamond for amino-acid determinations. 


\section{References}

Crawhall, J. C., and Watts, R. W. E. (1968). Cystinuria. American Journal of Medicine, 45, 736-755.

Dahlberg, P. J., Van Den Berg, C. J., Kurtz, S. B., Wilson, D. M., and Smith, L. H. (1977). Clinical features and management of cystinuria. Mayo Clinic Proceedings, 52, 533-542.

Macdonald, W. B., and Fellers, F. X. (1968). Quantitative measurement of cystine in urine. American Journal of Clinical Pathology, 49, 123-126.
Stokes, G. S., Potts, J. T., Jr, Lotz, M., and Bartter, F. C. (1968). New agent in treatment of cystinuria: N-acetylD-penicillamine. British Medical Journal, 1, 284-288.

Terhorst, B., and Stuhlsatz, H. W. (1975). Cystinsteintherapie mit Mercaptopropyonylglycin (MPG) (Thiola). Urologe; Ausgabe A, 14, 190-193.

Correspondence to Dr G. Ruysch van Dugteren, Renal Clinic, Red Cross War Memorial Children's Hospital, Rondebosch 7700, Cape Town, South Africa.

\title{
Takayasu's arteritis as a differential diagnosis of systemic juvenile chronic arthritis
}

\author{
EVE ROSSOR
}

Department of Paediatrics, Northwick Park Hospital, Harrow

SUMMARY After a series of throat infections an 8-year-old girl presented with fever and limb pain, initially diagnosed as juvenile chronic arthritis. Persistent symptoms led to further investigations when she was shown to have an arteritis affecting her aorta.

As there are no specific diagnostic tests for juvenile chronic arthritis (JCA), a remittent fever with a high swinging pattern associated with limb pain which continues for at least 2 weeks tends to be regarded as systemic JCA. However, a number of conditions, including various types of arteritis, can also produce these symptoms (McEnery, 1977).

\section{Case report}

An 8-year-old Yugoslavian girl was referred for advice on further management of what was thought to be systemic JCA of 18 months' duration. She was the only child of healthy unrelated parents and had been well until age 7 when, during a 4-month period, she had four episodes of pyrexia to $38^{\circ} \mathrm{C}$ associated with pharyngitis, which were treated with ampicillin. She subsequently developed fever with a temperature which characteristically reached $40^{\circ} \mathrm{C}$ daily between the afternoon and midnight, and then subsided spontaneously. She was generally unwell with considerable pain in her lower limbs, but no other signs or symptoms. Initial investigations showed ESR $97 \mathrm{~mm}$ in 1st hour, Hb $9.9 \mathrm{~g} / \mathrm{dl}$, WBC $13.0 \times 10^{9} / 1$, a hypercellular bone marrow, and ASO titre $<1 / 200$; blood cultures and all other investigations were negative. Skin and muscle biopsies were normal. She was given a 10-day course of lincomycin and gentamicin but her fever persisted; there was transient improvement with salicylate. Prednisolone was started at an initial dosage of $\mathbf{8 0}$ mg daily with pronounced improvement in ESR and fever. Both these parameters deteriorated when prednisolone was reduced to $10 \mathrm{mg}$ daily. She was continued on $10 \mathrm{mg}$ prednisolone daily and aspirin until referred here one year later.

When seen she still had an occasional fever, despite the prednisolone and aspirin. She was markedly cushingoid, and was on the 50th centile for height and the 90th centile for weight. There were no specific complaints but on direct questioning she did admit to some lower abdominal pain, discomfort in the calf muscles, and bleeding haemorrhoids. There were 4 small café-au-lait spots but no other cutaneous lesions. There was no lymphadenopathy, rash, hepatosplenomegaly, or arthritis. Her pulses were easily palpable in the arms and carotids, but no pulses were felt in the femoral arteries or in the legs, which were cold and hairless. No bruit was heard. Blood pressure was $130 / 80 \mathrm{mmHg}$ in the arms and, by Doppler, $90 / 0 \mathrm{mmHg}$ in the legs. All other systems were normal apart from first-degree haemorrhoids. Some days after examination it became obvious that the pain in the calves was, in fact, intermittent claudication as it appeared to be closely associated with the child walking some $300-400$ metres.

\section{Investigations}

ESR was raised at 57 rising to $88 \mathrm{~mm}$ in 1st hour on reduction of prednisolone, $\mathrm{Hb} 12 \cdot 2 \mathrm{~g} / \mathrm{dl}$, WBC 